\title{
Reynolds number effect on the velocity derivative flatness factor
}

\author{
M. Meldi ${ }^{1,2, \dagger}$, L. Djenidi ${ }^{3}$ and R. Antonia ${ }^{3}$ \\ ${ }^{1}$ Institut PPRIME, Department of Fluid Flow, Heat Transfer and Combustion, \\ CNRS - ENSMA - Université de Poitiers, UPR 3346, SP2MI - Téléport, \\ 211 Bd. Marie et Pierre Curie, B.P. 30179 F86962 Futuroscope Chasseneuil CEDEX, France \\ ${ }^{2}$ Aix-Marseille Univ., CNRS, Centrale Marseille, M2P2 Marseille, France \\ ${ }^{3}$ Discipline of Mechanical Engineering, School of Engineering, University of Newcastle, Newcastle, \\ 2308 NSW, Australia
}

This paper investigates the effect of a finite Reynolds number (FRN) on the flatness factor $(F)$ of the velocity derivative in decaying homogeneous isotropic turbulence by applying the eddy damped quasi-normal Markovian (EDQNM) method to calculate all terms in an analytic expression for $F$ (Djenidi et al., Phys. Fluids, vol. 29 (5), 2017b, 051702). These terms and hence $F$ become constant when the Taylor microscale Reynolds number, $R e_{\lambda}$ exceeds approximately $10^{4}$. For smaller values of $R e_{\lambda}, F$, like the skewness $-S$, increases with $R e_{\lambda}$; this behaviour is in quantitative agreement with experimental and direct numerical simulation data. These results indicate that one must first ensure that $R e_{\lambda}$ is large enough for the FRN effect to be negligibly small before the hypotheses of Kolmogorov (Dokl. Akad. Nauk SSSR, vol. 30, 1941a, pp. 301-305; Dokl. Akad. Nauk SSSR, vol. 32, 1941b, pp. 16-18; J. Fluid Mech., vol. 13, 1962, pp. 82-85) can be assessed unambiguously. An obvious implication is that results from experiments and direct numerical simulations for which $R e_{\lambda}$ is well below $10^{4}$ may not be immune from the FRN effect. Another implication is that a power-law increase of $F$ with respect to $R e_{\lambda}$, as suggested by the Kolmogorov 1962 theory, is not tenable when $R e_{\lambda}$ is large enough.

Key words: isotropic turbulence, turbulence modelling, turbulent flows

\section{Introduction}

At the 1961 international colloquium in Marseille and following Oboukhov (1962), Kolmogorov revised his 1941 theory or K41 (Kolmogorov 1941a), to address an issue first raised by Landau (see for example Landau \& Lifshitz 1987) shortly after K41 was published, namely the variation in the mean turbulent kinetic energy dissipation rate $\bar{\epsilon}$ induced by the large-scale motion. This revision, usually referred to as K62 (Kolmogorov 1962), is known as the 'refined' similarity hypothesis. For over almost 60 years and with a few exceptions (e.g. Kraichnan 1974; Qian 2000; Lundgren 2002; McComb 2014), the current dominant view is that the properties of small

$†$ Email address for correspondence: marcello.meldi@ensma.fr 
scale turbulence comply with K62. However, this view is currently being challenged (e.g. Antonia et al. 2015; Tang et al. 2015a,b; Antonia et al. 2017) on the basis that before the effect of intermittency can be adequately assessed, i.e. before one can unambiguously decide between K41 and K62 (a sine qua non requirement of both $\mathrm{K} 41$ and $\mathrm{K} 62$ is that the Reynolds number is very large) one must first ensure that the finite Reynolds number (FRN) effect is no longer active.

A study by Antonia \& Burattini (2006) (see also Zhou et al. 2000) showed that $\overline{(\delta u)^{3}}$ (the third-order moment of the longitudinal velocity increment $\delta u=(u(r)-u(0))$, where $r$ is the separation between two points separated in $x$, the longitudinal direction; the overbar denotes time and/or spatial averaging) approaches $-4 \bar{\epsilon} r / 5$ in the inertial range as the Taylor microscale Reynolds number, $R e_{\lambda}$, reaches large values in both decaying and forced homogeneous and isotropic turbulence (HIT). More recently, Tchoufag, Sagaut \& Cambon (2012) confirmed this trend by carrying out an eddy damped quasi-normal Markovian (EDQNM) calculation of the Lin equation. It should be stressed that $\overline{(\delta u)^{3}}=-4 \bar{\epsilon} r / 5$ is the so-called four-fifths law, derived by Kolmogorov (1941b) from the Navier-Stokes equation and thus is rigorous and exact when $\operatorname{Re}_{\lambda}$ is very large. Accordingly, these two studies reveal a clear Reynolds number effect (coined 'the finite Reynolds number or FRN effect'; see Qian 1997, 1999; Danaila et al. 1999; Lindborg 1999; Antonia \& Burattini 2006) otherwise ignored or, most likely, mistaken for the effect of the intermittency of $\epsilon$ on the small-scale motion. Further, recent studies (Antonia et al. 2015; Tang et al. 2015a,b) extended the assessment of the FRN effect on the skewness factor, $S$, of the longitudinal velocity derivative $(\partial u / \partial x)$ in several turbulent flows. These latter studies indicate that $S$ approaches the same constant, approximately -0.55 , in all flows investigated when $R e_{\lambda}$ increases. Approximately the same value (-0.53) was obtained in the EDQNM simulation of HIT by Meldi \& Sagaut (2013a) and much earlier by Qian (1994). This result, i.e. the approach toward an asymptotic constant value for $S$ with an increasing $R e_{\lambda}$, is consistent with $\mathrm{K} 41$, which predicts that $S$ is a universal constant; under the framework of $\mathrm{K} 62,-S \sim R e_{\lambda}^{\alpha}$, where $\alpha$ is a small positive real number $(0 \leqslant \alpha \leqslant 1)$.

It is worth stressing that despite their conflicting predictions, K41 and K62 assume that the Reynolds number is very large and local isotropy is satisfied (following a long energy cascade process). These two requirements must be met before one can test these theories. The second requirement would exclude certain flows or flow regions, for example, and as noted by Kolmogorov, those in the vicinity of boundaries. In this context, it is somewhat surprising that data obtained in the atmospheric shear layer (ASL) (see Sreenivasan \& Antonia 1997) where $R e_{\lambda}$ can be large were used to test K41 and K62. Lack of information on the ASL layer thickness $\delta$ makes it very difficult to estimate $y / \delta$ ( $y$ is the height above the ground or ocean surface). Accordingly, one cannot rule out that the ASL data as reported in Sreenivasan \& Antonia (1997) and often measured at only a few metres above the surface are contaminated by the near-surface effects (Djenidi et al. 2017a; Meldi \& Sagaut 2017). ASL data are not immune from the FRN effect (as noted in Sreenivasan \& Antonia 1997) as well as the influence of the strong shear. In addition, another open question is whether the threshold value for $R e_{\lambda}$ for which FRN effects can be safely neglected is the same for every flow statistical quantity. Numerical results by Meldi \& Sagaut (2013a) seem to indicate that this value increases with higher-order statistics. Tang et al. (2017) who investigated the effect of the FRN effect in the scaling range exponent $\zeta_{n}\left(\overline{(\delta u)^{n}} \sim r^{\zeta_{n}}\right)$ showed that the magnitude of $\zeta_{n}$ increases, strictly the maximum value of $\left(\overline{(\delta u)^{n}} r^{-\zeta_{n}}\right)$, as $R e_{\lambda}$ increases and the rate of increase depends on the order $n$. 
In the present study, we investigate, via EDQNM calculations, the FRN effect in freely decaying HIT. Particular emphasis is on the behaviour of the flatness factor, $F$, of $\partial u / \partial x$ over a very large range for $R e_{\lambda}$. A similar EDQNM analysis on the velocity derivative skewness factor, $S$, was reported in Meldi \& Sagaut (2013a). Since, conversely to $S, F$ cannot be directly computed from the EDQNM calculations, the FRN effect on $F$ is investigated by assessing the FRN effect on a new analytic expression for $F$, rigorously derived from the Navier-Stokes equations (Djenidi et al. $2017 b$ ) for HIT. The novelty of the present contribution lies in the ability to assess the FRN effect when $R e_{\lambda}$ varies from 200 to $5 \times 10^{5}$. This is possible due to the minimal computational resources required by the EDQNM simulations. This range of $R e_{\lambda}$, which exceeds three decades, allows for an unambiguous identification of the FRN effect on both $S$ and $F$, with the expectation that FRN effects identified via EDQNM are at least comparable if not identical to the behaviour described by the Navier-Stokes equations. Such an extended range of $R e_{\lambda}$ cannot be reached, either experimentally or with conventional direct numerical simulation (DNS).

\section{Flatness factor of $\partial u / \partial x$}

\subsection{A rigorous analytic expression}

The flatness factor of $\partial u / \partial x$ is defined as

$$
F=\frac{\overline{(\partial u / \partial x)^{4}}}{{\overline{(\partial u / \partial x)^{2}}}^{2}}
$$

However, starting with the equation for $\overline{(\delta u)^{3}}$ (as first written by Hill (2001)), applying self-similarity (or scale invariance) and taking the limit $r \rightarrow 0$, Djenidi et al. (2017b) derived the following analytic equation for $F$ :

$$
F+\frac{10}{3} \gamma_{1}-\frac{20}{3} \frac{\gamma_{2}}{R e_{\lambda}} \simeq-\alpha \frac{S}{R e_{\lambda}}
$$

where $\gamma_{1}=\left(\lambda^{4} / u^{\prime 4}\right) \overline{(\partial u / \partial x)^{2}\left(\partial^{2} p / \partial x^{2}\right)}, \quad \gamma_{2}=\left(\lambda^{5} / u^{\prime 3}\right) \overline{\tilde{\epsilon}_{H I T}\left(\partial^{3} u / \partial x^{3}\right)}$ with $\tilde{\epsilon}_{H I T}=(7 / 6)$ $(\partial u / \partial x)^{2}-(2 / 3)(\partial u / \partial y)^{2}(p$ is the kinematic pressure fluctuation) and $\alpha$ is a numerical (dimensionless) constant. In this expression $\lambda$ and $u^{\prime}$ were used as scaling variables. If the Kolmogorov scales are used, equation (2.2) can be replaced by

$$
F+750\left[\gamma_{1, K}-4 \gamma_{2, K}\right] \simeq-\frac{100}{3} \frac{S}{R e_{\lambda}},
$$

where the subscript $K$ represents Kolmogorov normalisation. The second and third terms on the left-hand side of (2.2) or (2.3) represent the actions of pressure and viscosity, respectively. Noting that $S / R e_{\lambda} \rightarrow 0$, irrespectively of whether $S$ is constant or varies like $\operatorname{Re}_{\lambda}^{\alpha}(0 \leqslant \alpha \leqslant 1)$, equation (2.2) can be written as

$$
F \simeq-\frac{10}{3} \gamma_{1}+\frac{20}{3} \frac{\gamma_{2}}{R e_{\lambda}}
$$

for large $R e_{\lambda}$. In Djenidi et al. (2017b), the second term on the right-hand side of (2.4) was assumed to be negligible when $R e_{\lambda}$ becomes very large. Since there is no a priori justification for this, it has been retained. 
It is important to stress that (2.2), a rigorous model-free expression for $F$, is derived from the Navier-Stokes equations under the assumption of self-similarity. Note though that the presence of the pressure term makes it difficult, if not impossible, to test this expression experimentally. One must resort to direct numerical simulations (DNS). To date, the highest $R e_{\lambda}$ reached by DNS in forced HIT (Ishihara et al. 2007) is approximately 1150 . The maximum $R e_{\lambda}$ for DNS of freely decaying HIT is smaller, because of lingering effects of initial conditions and confinement effects (Meldi \& Sagaut 2017), thus limiting the range of investigation of $R e_{\lambda}$. On the other hand, $R e_{\lambda}$ of order $10^{5}$ can be achieved with EDQNM simulations of decaying HIT (Meldi \& Sagaut 2013a). However, this model calculates the time evolution of the energy spectrum $E$ in Fourier transform space, so that the terms of (2.4) cannot be directly obtained. Therefore, we use an analysis based on dimensional arguments on the statistical quantities available via EDQNM, which allows us to qualitatively assess their Re-dependence. This dimensional analysis is consistent with the assumption of self-similarity used to derive (2.2).

\subsection{Dimensional arguments}

While the definitions of the coefficients $\gamma_{1}$ and $\gamma_{2}$ are clear in physical space, their formulation in a spectral frame of reference is elusive. The second-order link between statistics in the physical and spectral spaces is obtained by comparing the Kármán-Howarth equation with the Lin equation. For third-order statistics, the link is provided using the time evolution equation for the energy dissipation rate $\bar{\epsilon}$ in the spectral domain (Meldi \& Sagaut 2013a) and the vorticity equation in physical space. Restricting the analysis to purely isotropic turbulence, both equations exhibit a destruction term which is determined by a spectral integration including a correct dimensional combination of the wavenumber $k$ and the energy spectrum $E(k, t)$. In particular, the expressions are $\bar{\epsilon} \propto \overline{(\partial u / \partial x)(\partial u / \partial x)} \propto \int k^{2} E \mathrm{~d} k$ and $G\left(\bar{\epsilon}^{2} / \mathcal{K}\right) \propto \overline{\left(\partial^{2} u / \partial x^{2}\right)\left(\partial^{2} u / \partial x^{2}\right)} \propto \int k^{4} E \mathrm{~d} k$ for the energy dissipation rate and the palinstrophy, respectively. A corresponding relation between physical and spectral spaces is missing for fourth-order statistics. However, the dimensions of the terms in the equation for $\overline{(\delta u)^{3}}$ used to derived expression (2.2) are expressed in terms of the metre $m$ and second $s$. Further, these terms can be represented spectrally. For example, let us consider the pressure term and follow Djenidi et al. (2017b) who, after applying the Cauchy-Schwarz theorem, obtained the following expression:

$$
\left|\overline{\left(\frac{\partial u}{\partial x}\right)^{2} \frac{\partial^{2} p}{\partial x^{2}}}\right| \leqslant \overline{\left(\frac{\partial u}{\partial x}\right)^{4}}{\overline{\left(\frac{\partial^{2} p}{\partial x^{2}}\right)^{2}}}^{1 / 2}
$$

which finally leads to (Djenidi et al. 2017b)

$$
\left|\gamma_{1}\right|=\frac{\lambda^{4}}{u^{\prime 4}}\left|\overline{\left(\frac{\partial u}{\partial x}\right)^{2}\left(\frac{\partial^{2} p}{\partial x^{2}}\right)}\right| \leqslant F^{1 / 2}\left(\int_{0}^{\infty} k^{* 4} E_{p}^{*}\left(k^{*}\right) \mathrm{d} k^{*}\right)^{1 / 2},
$$

where the symbol $*$ represents normalisation using $\lambda$ and $u^{\prime}$ as scaling variables. Considering that $p$ can be easily expressed in terms of $u$, one can expect that $\gamma_{1}$ may be expressed in terms of $k$ and $E(k)$, as will be shown below. Since both terms on the right-hand side of (2.4) must have the same dimensions, then $\gamma_{2}$ can, like $\gamma_{1}$, also be expressed in the spectral domain via dimensional arguments in terms of $k$ and 
$E(k)$. For the sake of simplicity we assume that, under isotropy, $\overline{(\partial u / \partial y)_{2}}=2 \overline{(\partial u / \partial x)_{2}}$ and

$$
\overline{\left(\frac{7}{6}\left(\frac{\partial u}{\partial x}\right)^{2}-\frac{2}{3}\left(\frac{\partial u}{\partial y}\right)^{2}\right) \frac{\partial^{3} u}{\partial x^{3}}} \sim \overline{\left(\frac{\partial u}{\partial x}\right)^{2} \frac{\partial^{3} u}{\partial x^{3}}} .
$$

We can now use dimensional arguments to derive $\gamma_{1}$ and $\gamma_{2}$. First, we write $\gamma_{1} \propto$ $\overline{(\partial u / \partial x)^{2}\left(\partial^{2} p / \partial x^{2}\right)} \approx \overline{(\partial u / \partial x)^{4}}=\xi_{1}$, where we used the relation between $p$ and $u$. We write $\gamma_{1} \propto \xi_{1}$, and note that $\overline{(\partial u / \partial x)^{4}}=\overline{(\partial u / \partial x)^{2}(\partial u / \partial x)^{2}}$ exhibits similarities with the spectral transforms of $\epsilon$ and $G$. This term has dimensions of $\left[m^{0} s^{-4}\right]$. Starting from the assumption that this term depends only on $k$ and $E$, dimensional arguments lead to:

$$
\overline{\left(\frac{\partial u}{\partial x}\right)^{4}} \rightarrow \xi_{1}=a_{\xi_{1}} \int_{0}^{+\infty} k^{5} E^{2} \mathrm{~d} k .
$$

It should be stressed that we have investigated a number of different combinations of $k$ and $E$ complying with $\left[m^{0} s^{-4}\right]$, including the dimensional quantities $\lambda^{-1} \int_{0}^{\infty} k^{4} E^{2} \mathrm{~d} k$ and $\int_{0}^{\infty} k^{4} E_{p}(k) \mathrm{d} k$. The high Reynolds number behaviour of all these quantities, which is the subject of the present investigation, is identical.

A similar approach is employed to derive a spectral expression for the coefficient $\gamma_{2}$. The starting point is the quantity $\overline{(\partial u / \partial x)^{2}\left(\partial^{3} u / \partial x^{3}\right)}$, whose dimension is $\left[m^{-2} s^{-3}\right]$. Again, dimensional arguments based on $k$ and $E$ yields:

$$
\overline{\left(\frac{\partial u}{\partial x}\right)^{2} \frac{\partial^{3} u}{\partial x^{3}}} \rightarrow \xi_{2}=a_{\xi_{2}} \int_{0}^{+\infty} k^{11 / 2} E^{3 / 2} \mathrm{~d} k .
$$

Finally, a third term is investigated. While it is not directly connected with the terms in (2.4), it is introduced to allow us to define a new invariant. This term can be obtained from the homogeneity condition:

$$
\frac{\partial}{\partial x} \overline{\left(\frac{\partial u}{\partial x}\right)^{2}\left(\frac{\partial^{2} u}{\partial x^{2}}\right)}=0
$$

which implies that $\overline{(\partial u / \partial x)^{2}\left(\partial^{3} u / \partial x^{3}\right)} \propto \overline{\left(\partial^{2} u / \partial x^{2}\right)^{2}(\partial u / \partial x)}$. Taking into account the exact relation $\overline{(\partial u / \partial x)^{3}}=\overline{(\partial u / \partial x)^{2}(\partial u / \partial x)} \rightarrow \int_{0}^{+\infty} k^{2} T \mathrm{~d} k$ and since the square of $(\partial u / \partial x)^{2}$ is linked to the square of the second derivative $\left(\partial^{2} u / \partial x^{2}\right)^{2}$ via a $k^{2}$ term in spectral space (Pope 2000), the following relation is obtained:

$$
\overline{\left(\frac{\partial^{2} u}{\partial x^{2}}\right)^{2} \frac{\partial u}{\partial x}} \rightarrow \xi_{3}=a_{\xi_{3}} \int_{0}^{+\infty} k^{4} T \mathrm{~d} k .
$$

Thus, we can introduce a new coefficient $\gamma_{3} \propto \xi_{3}$, whose behaviour will be investigated in $\S 4.4$. The expressions for $\xi_{1}$ and $\xi_{2}$ allow us to ascertain the Reynolds number behaviour of the flatness expression coefficients $\gamma_{1}, \gamma_{2}$. As will be seen later, the coefficient $\gamma_{3}$ allows us to define and investigate a new invariant. It can be defined similarly to $\gamma_{2}$ because of dimensional analogy between $\xi_{2}$ and $\xi_{3}$. Thus, finally, the results of the dimensional argument analysis are:

$$
\gamma_{1}=-\frac{\lambda^{4}}{\left(\frac{2}{3} \mathcal{K}\right)^{2}} \xi_{1}, \quad \gamma_{2}=\frac{\lambda^{5}}{\left(\frac{2}{3} \mathcal{K}\right)^{3 / 2}} \xi_{2}, \quad \gamma_{3}=\frac{\lambda^{5}}{\left(\frac{2}{3} \mathcal{K}\right)^{3 / 2}} \xi_{3},
$$


where $\mathcal{K}$ is the turbulent kinetic energy. Interestingly, Tang et al. (2018) recently showed that the term $\gamma_{2} / R e_{\lambda}$ in (2.4) becomes negligible as $R e_{\lambda}$ increases. Thus, for large $R e_{\lambda}$, equation (2.4) leads to $F \approx \gamma_{1}$, and the following approximation can be obtained (Djenidi et al. 2017b):

$$
F \leqslant \int_{0}^{\infty} k^{* 4} E_{p}^{*}\left(k^{*}\right) \mathrm{d} k^{*}
$$

Since the right-hand side of this expression can be explicitly calculated using the EDQNM model (Meldi \& Sagaut 2013b), we will also assess its $R e_{\lambda}$-behaviour.

\section{The EDQNM model}

The eddy damped quasi-normal Markovian (EDQNM) model (Orszag 1970; Lesieur 1997; Sagaut \& Cambon 2018) is introduced in the present section. The model numerically resolves a discretisation in the spectral space (wavenumber $k$ ) of the Lin equation, which describes the time evolution of the energy spectrum $E(k, t)$ :

$$
\frac{\partial E(k, t)}{\partial t}+2 v k^{2} E(k, t)=T(k, t)
$$

where $v$ is the molecular viscosity of the fluid and $T(k, t)$ is the nonlinear energy transfer. The EDQNM closure acts on the evolution equation of $T$ via two important approximations:

(i) the fourth-order cumulants, which represent the deviation of the velocity derivative pdf from a Gaussian distribution, are approximated using an eddy damping term (eddy damping hypothesis);

(ii) time integration is simplified via a Markovianisation procedure.

A closed expression for the nonlinear energy transfer $T(k, t)$ is obtained via shell integration of radius $k$ :

$$
T_{E D Q N M}=\int_{p+q=k} \Theta_{k p q} g E(q, t)\left[E(p, t) p k^{2}-E(k, t) p^{3}\right] \frac{\mathrm{d} p \mathrm{~d} q}{p q},
$$

where $\Theta_{k p q}$ is a characteristic time resulting from the Markovian approximation and $g$ is a geometric function.

The two underlying hypotheses used to obtain the closed expression (3.2) for the nonlinear energy transfer $T$ are well verified and their effect is expected to be very small, if not negligible, when compared with FRN effects which are presently investigated. Indeed, the Markovianisation hypothesis is an accurate approximation when a clear separation is observed between the turbulent characteristic time scale and the turnover time scale of the physical quantities investigated. The present analysis clearly satisfies this condition since the physical quantities investigated are intimately associated with the dissipative scales, which, considering the large values of $R e_{\lambda}$ used, are well separated from the large scales. Further, the eddy damping hypothesis provides an accurate representation of fourth-order cumulants. This can be verified by comparing the present predicted velocity derivative skewness $S$ with the experimental data (see figure 1). The data reported in figure 1 have been collected from the relatively recent reappraisal of $S$ in various turbulent flows (Antonia et al. 2015), which includes a comprehensive overview of results reported in the literature; 


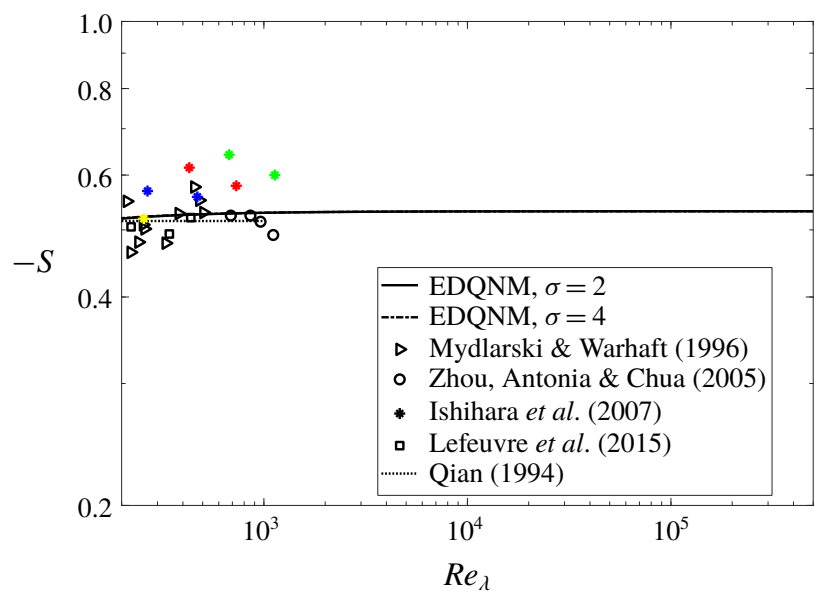

FIGURE 1. (Colour online) Velocity derivative skewness $S$ variation with $R e_{\lambda}$. The EDQNM prediction is compared with data in the literature (Antonia et al. 2015). The present curves for $\sigma=2$ and 4 are practically indistinguishable. For the Ishihara data, the colours represent different mesh resolutions; yellow: $512^{3}$, blue: $1024^{3}$, red: $2048^{3}$, green: $4096^{3}$.

only a sample, for which $R e_{\lambda} \geqslant 200$, is shown in the figure (Qian 1994; Mydlarski \& Warhaft 1996; Zhou, Antonia \& Chua 2005; Lefeuvre, Djenidi \& Antonia 2015). Accounting for the scatter in the experimental data due to measurement uncertainties, Antonia et al. (2015) showed that as $R e_{\lambda}$ increases, $-S$ first increases before becoming $R e_{\lambda}$-independent with a value around 0.53 ; they showed that while the approach rate toward this value differs from flow to flow, the value is approximately well reached for all flows when $R e_{\lambda} \simeq 200-300$. The present prediction of the $R e_{\lambda}$ behaviour of $-S$ is in agreement with the observation of Antonia et al. (2015) and the prediction of Qian (1994). The figure also shows all values of $-S$ from the DNS data of Ishihara et al. (2007) computed with different mesh resolutions and two wavenumber truncations $\left(k_{\max } \eta=1\right.$ and 2$)$. It is difficult to draw a definitive conclusion as to the actual behaviour of $-S$ obtained from these DNS data as $R e_{\lambda}$ increases, due to the variability of the results associated with the choice of the wavenumber truncation. One may argue that the data indicate a slight increases with $R e_{\lambda}$, which Antonia et al. (2015) associated with the fact that the Kolmogorov normalised spectra do not conform with Kolmogorov scaling in the dissipative range. Further, it should be remarked that Ishihara et al. (2007) concluded that it is possible that as $\operatorname{Re}_{\lambda} \rightarrow \infty, S$ approaches a constant independent of $R e_{\lambda}$, but the approach may be slow; this remark is certainly consistent with the trend shown by all the other data. The reader may consult Antonia et al. (2015) for a full account on the behaviour of $-S$ with $R e_{\lambda}$. The message conveyed by figure 1 is that the EDQNM hypotheses have a negligible impact on the turbulence statistics, at least a much weaker impact than that of the FRN effects.

The model has features which are useful when analysing the coefficients in (2.12):

(i) It allows for the analysis of freely decaying isotropic turbulence at very high $R e_{\lambda}$, approximately three orders of magnitude larger than can be realistically achieved using DNS. In addition, continuous screening over several decades for $\operatorname{Re}_{\lambda}$ can be performed excluding confinement effects, which play a major role in the 
time evolution of turbulence statistics (Meldi \& Sagaut 2017). Previous analyses reported in the literature by Meldi \& Sagaut (2013a) indicate that the EDQNM provides a correct representation of asymptotic high- $R e_{\lambda}$ and low- $R e_{\lambda}$ regimes. The comparison of these results with a large survey of experiments (Djenidi, Kamruzzaman \& Antonia 2015) also shows that the EDQNM transition between the two regimes is consistent with physical flow observation. For these reasons, the representation of FRN effects via EDQNM should be at least qualitatively comparable with the exact quantification obtainable via Navier-Stokes equations.

(ii) It does not include an intermittency model. First, there is no definitive intermittency model that can be used with confidence. Second, it is yet to be shown that one is actually required; no such model is required to derive (2.2). In addition, both the Lin equation and the Navier-Stokes equations are free of any intermittency model; intermittency is accounted for naturally in these equations. Accordingly, excluding an intermittency model from the EDQNM simulation is consistent with the derivation of (2.2) and thus allows us to focus on the finite Reynolds number (FRN) effects on free decaying HIT up to very high Reynolds numbers. We recall here that, as indicated by Grossmann \& Lohse (1994), an understanding of intermittency has to come from the Navier-Stokes equations. Finally, and quite importantly, figure 1, which shows that the present EDQNM simulation results of $-S$ are consistent with experimental, theoretical and DNS data, justifies the non-inclusion of an intermittency model in the EDQNM simulations.

A similar analysis of the velocity derivative flatness factor was carried out by Qian (1986) using a turbulence model based on a variational approach (Qian 1982) (using this approach Qian (1994) studied the dependence of $S$ with $R e_{\lambda}$ ). In this model an approximate solution of the stationary Liouville equation is derived by a perturbation method based on a Langevin-Fokker-Planck model. In this framework, the nonlinear interactions are simplified via a dynamic damping coefficient, $\eta$, and a random force described by a uniform probability distribution function. The parameter $\eta$ is used as a control variable which is optimised to minimise the error of the perturbation solution. The model based on the variational approach used by Qian (1982) is different to the EDQNM model and is limited to stationary turbulence; the solution is obtained as a perturbation of a stationary problem while the EDQNM closure can be used to investigate freely decaying HIT as well as forced HIT regimes (Meldi 2016). Nevertheless, the expressions for the nonlinear energy transfer $T$ obtained via the two models are similar. One example which illustrates how the EDQNM prediction of $T$ appropriately captures the FRN effects in HIT is given in the work of Tchoufag et al. (2012). They investigated the variation of $\overline{(\delta u)^{3}}$ with $R e_{\lambda}$. Their results are in very good agreement with those of Antonia \& Burattini (2006), inferred from the Kármán-Howarth equation and a model for the second-order velocity structure function. This agreement increases our confidence in the ability of EQDNM to estimate the FRN effects correctly.

\section{Results}

\subsection{Test case}

The test case is now described. EDQNM calculations are initialised via an energy spectrum functional form inspired by proposals by Pope (2000) and Meyers \& Meneveau (2008):

$$
E(k)=C_{k} \varepsilon^{2 / 3} k^{-5 / 3} f_{L}(k L) f_{\eta}(k \eta),
$$



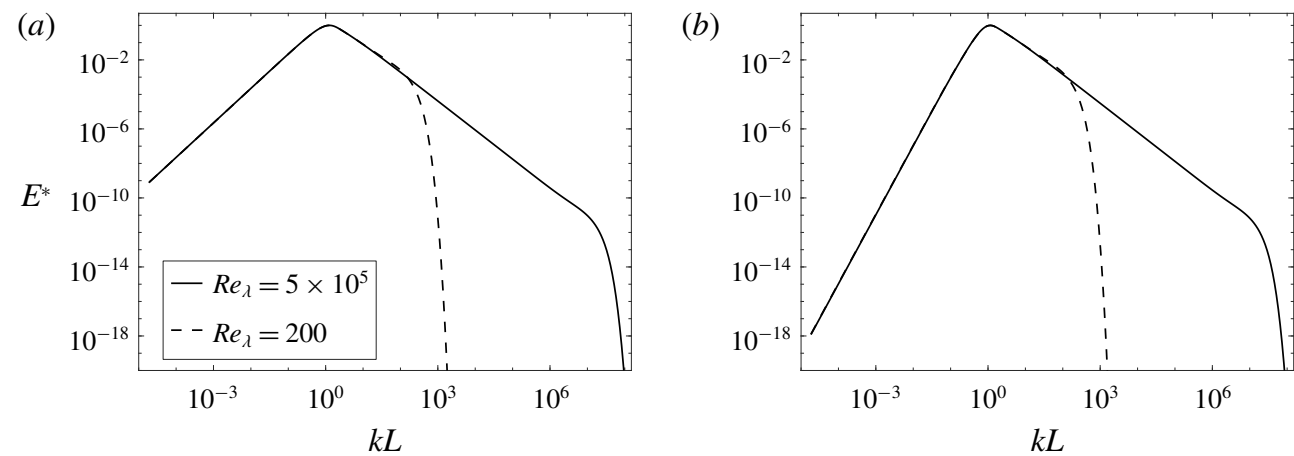

FIgURE 2. Normalised energy spectra $E^{*}=E / \max (E)$ characterising the initial state and final state of the observation window of the analysis. The spectra are shown for the two types of turbulence, namely (a) Saffman turbulence and (b) Batchelor turbulence.

with

$$
f_{L}(k L)=\left(\frac{k L}{\left[(k L)^{1.5}+c_{L}\right]^{1 / 1.5}}\right)^{5 / 3+\sigma}, \quad f_{\eta}(k \eta)=\exp \left(-\beta\left(\left[(k \eta)^{4}+c_{\eta}^{4}\right]^{1 / 4}-c_{\eta}\right)\right), \quad(4.2 a, b)
$$

where the free coefficients have been set to $c_{\eta}=0.4, \beta=5.3 ; c_{L}$ has been chosen in order to obtain $L(0)=1$. Two calculations have been performed for values of the parameter $\sigma=2,4$. This parameter controls the shape of the energy spectrum at large scales, and the values chosen correspond to the well-known cases of Saffman turbulence ( $\sigma=2$; Saffman 1967) and Batchelor turbulence ( $\sigma=4$, Batchelor 1948). The initial Reynolds number has been set to $R e_{\lambda}=5 \times 10^{5}$. During early stages of decay, the energy spectrum exhibits an anomalous evolution, which is associated with the approximated functional form prescribed for $t=0$. This is the reason why an increase of the Reynolds number is observed up to $R e_{\lambda}=8 \times 10^{5}$ for $t \approx t_{0}$, where $t_{0}=$ $\mathcal{K}(0) / \bar{\epsilon}(0)$ is the initial turnover time. After this first phase, the statistics progressively lose memory of the initial condition and a classical power-law decay is observed (Comte-Bellot \& Corrsin 1966; Meldi \& Sagaut 2012). For the present analysis, data are sampled in the range $5 \times 10^{5} \geqslant R e_{\lambda} \geqslant 200$. Initial and final spectra in the range of analysis are shown in figure 2 , while the decay of turbulent kinetic energy $\mathcal{K}$ and the associated power-law exponent $n_{\mathcal{K}}$ are shown in figure 3 . The results clearly show a power-law decay of $\mathcal{K}$, and the apparent faster energy decay of Saffman turbulence is simply related to a much slower decay of the Reynolds number $R e_{\lambda}$ in this case. In fact, the determination via polynomial fitting of the power-law exponents $n_{\mathcal{K}}$ which govern the relation $\mathcal{K}(t) \propto\left(t / t_{0}\right)^{n_{\mathcal{K}}}$ indicates that classical results obtained using ComteBellot/Corrsin formulae (Comte-Bellot \& Corrsin 1966) are obtained within a $5 \%$ tolerance. The analytic formulae predict a value of $n_{\mathcal{K}}=-2(\sigma-a+1) /(\sigma-a+3)$ where the coefficient $a$ represents a correction term due to non-local interaction and is equal to $a=0$ for Saffman turbulence and $a \approx 0.52$ for Batchelor turbulence (Meldi $\&$ Sagaut 2012). The present analysis encompasses more than three decades of $R e_{\lambda}$, in which a significant evolution of the statistical quantities is observed. In particular, the integral length scale $L$ exhibits an increase of 14 decades for Saffman turbulence and of 6 decades for Batchelor turbulence over the range investigated. In order to exclude confinement effects, the adaptive version of the EDQNM model proposed by Meldi \& Sagaut (2014) has been used. In this case, a fixed large-scale resolution 

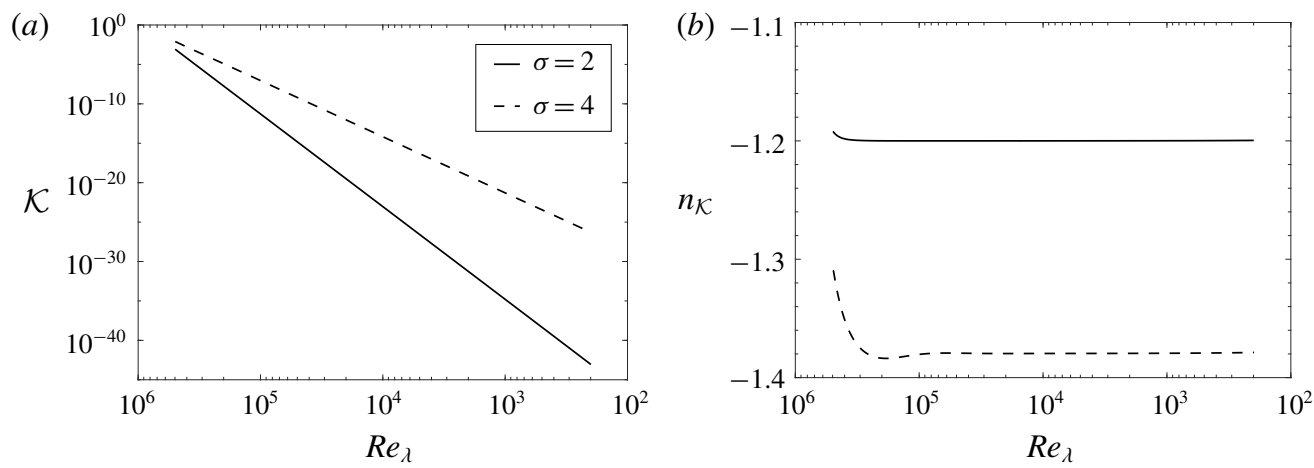

FIgURE 3. (a) Evolution of the turbulent kinetic energy $\mathcal{K}$ for the two cases investigated over the observation window. (b) Power-law exponents $n_{\mathcal{K}}$ characterising the power-law decay of $\mathcal{K}$.

of $k_{L}(t) / k_{1}(t)=5 \times 10^{4}$ has been imposed. Here $k_{L}(t)=L^{-1}(t)$ is the wavenumber associated with the integral length scale, while $k_{1}(t)$ is the smallest resolved mode. Equivalently, a small-scale resolution of $\eta(t) k_{M A X}(t)>10$ has been imposed, where $k_{M A X}(t)$ is the largest resolved mode. This implies that a resolution equivalent to at least $0.1 \eta$ is obtained. The mesh elements are distributed following a geometrical progression of ratio $r=1.12$, which grants 20 mesh elements over each decade of the spectral mesh.

In summary, the use of the adaptive EDQNM model allows for the analysis of very high Reynolds number configurations over several decades of evolution time, naturally excluding confinement effects. Thus, the EDQNM result can provide new elements for the analysis of experiments and DNS data; this is based on the expectation that the quantification of FRN effects in EDQNM should be similar if not identical to the behaviour derived from the Navier-Stokes equations.

\subsection{Finite Reynolds number effects on $\gamma_{i}$ coefficients}

The analysis of the coefficients derived via EDQNM calculations is now performed. The $R e_{\lambda}$-variation of these coefficients is shown in figure 4 . The coefficient $\gamma_{1 P}=\int_{0}^{\infty} k^{* 4} E_{p}^{*}\left(k^{*}\right) \mathrm{d} k^{*}$, reported in figure $4(a)$, is investigated first. It is normalised over its value for $\operatorname{Re}_{\lambda}=5 \times 10^{5}$. This coefficient may represent an upper limit for $F$ (see (2.13) and Tang et al. 2018) and thus its $R e_{\lambda}$-behaviour is worthwhile assessing. It first increases with $R e_{\lambda}$ before becoming practically constant for $R e_{\lambda} \gtrsim 10^{4}$, which suggests that $F$ must be bounded if (2.13) is valid. In this case, the behaviour $F \propto R e_{\lambda}^{\alpha}$, $\alpha>0$, which can be adequate for low to moderate $R e_{\lambda}$, is not tenable at very large $R e_{\lambda}$. This result (i.e. $\gamma_{1 P}$ becomes $R e_{\lambda}$-independent only when $R e_{\lambda}$ reaches a value of the order $10^{4}$ ) is consistent with increasing evidence in the literature showing that the higher the statistical moment, the higher the threshold of the Reynolds number beyond which the FRN effects become negligible. Next, the parameter $\gamma_{1}$ is shown in figure $4(b)$. Note that the value of $a_{\xi_{1}}$ is fixed so that $10 / 3\left|\gamma_{1}\right|=1$ for $\operatorname{Re}_{\lambda}=5 \times 10^{5}$. Similarly to $\gamma_{1 P}, \gamma_{1}$ becomes constant when $R e_{\lambda} \gtrsim 10^{4}$ while exhibiting an inversed trend to that of $\gamma_{1 P}$ for lower $R e_{\lambda}$. It decreases by approximately 2-3\% over the range of $R e_{\lambda}$ considered, which is larger than the $0.5 \%$ variation observed for $\gamma_{1 P}$ over the same $R e_{\lambda}$ range. The rates of variation of $\gamma_{1}$ and $\gamma_{1 P}$ with $R e_{\lambda}$ are sensitive 

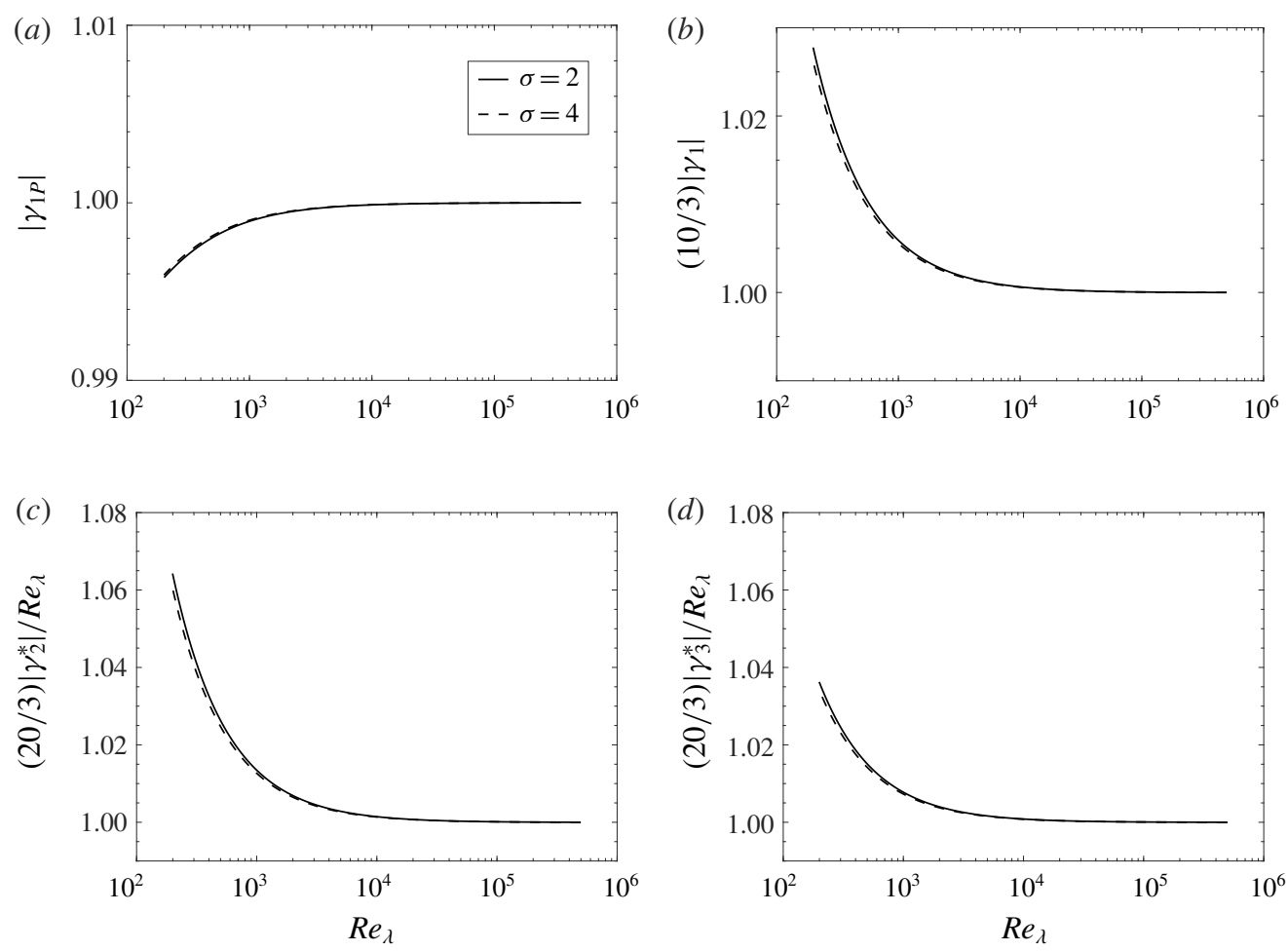

FIGURE 4. Evolution of the coefficients determined via EDQNM calculations. The coefficients $(a) \gamma_{1 P},(b) \gamma_{1},(c) \gamma_{2} / R e_{\lambda}$ and $(d) \gamma_{3} / R e_{\lambda}$ are shown for Saffman turbulence $(\sigma=2)$ and Batchelor turbulence $(\sigma=4)$.

to the behaviour of the large scales, which is magnified at lower Reynolds numbers because of the progressive reduction in the scale separation with a decreasing $R e_{\lambda}$. Similar conclusions can be drawn for the normalised parameters $\gamma_{2}^{*} / \operatorname{Re}_{\lambda}$ and $\gamma_{3}^{*} / \operatorname{Re}_{\lambda}$, which are shown in figures $4(c)$ and $4(d)$, respectively. In this case, the values of the parameters $a_{\xi_{2}}$ and $a_{\xi_{3}}$ have been selected so that (20/3)|$\gamma_{2}^{*}\left|/ \operatorname{Re}_{\lambda}=(20 / 3)\right| \gamma_{3}^{*} \mid / \operatorname{Re}_{\lambda}=1$ for $R e_{\lambda}=5 \times 10^{5}$. However, it appears that the two parameters are more sensitive than $\gamma_{1}$ to the FRN effects. Indeed, $\gamma_{2} / R e_{\lambda}$ and $\gamma_{3} / R e_{\lambda}$ varies by approximately $6 \%$ and $4 \%$, respectively. Interestingly, the EDQNM prediction for $\gamma_{2} / \operatorname{Re}_{\lambda}$ at low $\operatorname{Re}_{\lambda}$ (i.e. it decreases as $R e_{\lambda}$ increases) is consistent with that observed in experimental data for the various flow configurations reported in Tang et al. (2018). The latter authors observe that $\gamma_{2} / R e_{\lambda}$ approaches, relatively quickly, a very small (at least negligible when compared to $\gamma_{1}$ ) constant value as $R e_{\lambda}$ increases.

An important observation that stems from the above results is that FRN effects play a non-negligible role at low and moderate Reynolds numbers, but becomes negligible at high Reynolds numbers, much higher than the limit presently achievable in experimental facilities or with direct numerical simulation (DNS). One could then argue that the behaviour of both $S$ and $F$ commonly associated with the intermittency effect simply reflects the FRN effects.

\subsection{Estimation of the velocity derivative flatness factor $F$}

The results of $\S 4.2$ indicated that the parameters $\gamma_{i}$, which are correlated with features of the energy spectrum calculated via EDQNM, exhibit an asymptotic finite 
limit for $\operatorname{Re} \lambda \geqslant 104$. In the present section, we use these results to analyse the sensitivity of $F$ to $R e_{\lambda}$. The analysis is performed using two different strategies:

(i) we compute the analytic formulae for $F$ proposed by Qian (1986) using the EDQNM energy spectra;

(ii) the pre-multiplying coefficients $a_{\xi_{i}}$ in (2.8) and (2.9) are optimised in order to provide an EDQNM estimate of $F=-(10 / 3) \gamma_{1}+(20 / 3)\left(\gamma_{2} / R e_{\lambda}\right)$.

Strategy (i)

Qian (1986) obtained an expression for the velocity derivative flatness factor from a perturbation of the Liouville equation, which can be written as

$$
F=F^{(0)}+F^{(1)}+F^{(2)}+\cdots=3+F^{(2)}+\cdots
$$

After a complex manipulation, a closed expression for $F^{(2)}$ is obtained as a ninedimensional integral in the wavevector space $[\boldsymbol{p}, \boldsymbol{r}, \boldsymbol{s}]$ :

$$
\begin{aligned}
F^{(2)}= & 5400\left(\frac{v}{\epsilon}\right)^{2} \iiint \mathrm{d} \boldsymbol{p} \mathrm{d} \boldsymbol{r} \mathrm{d} \boldsymbol{s} \bar{G}(\boldsymbol{k}, \boldsymbol{p}, \boldsymbol{r}, \boldsymbol{s}, \boldsymbol{t}) \bar{q}(p) \bar{q}(r) \bar{q}(s) \\
& \times((\bar{\eta}(t)+\bar{\eta}(r)+\bar{\eta}(s)) \times(\bar{\eta}(k)+\bar{\eta}(p)+\bar{\eta}(r)+\bar{\eta}(s)))^{-1},
\end{aligned}
$$

where $\bar{G}$ is a geometric factor and $\boldsymbol{t}=\boldsymbol{r}+\boldsymbol{s}, \boldsymbol{k}=\boldsymbol{t}-\boldsymbol{p}$. Qian (1986) provided the following expressions for $\bar{q}(k)$ and $\bar{\eta}(k)$ in order to estimate the integral in (4.4):

$$
\begin{gathered}
\bar{\eta}(k)=0.268 \epsilon^{1 / 3} k^{2 / 3}\left(1+3.73(k \eta)^{4 / 3}\right), \\
\bar{q}(k)=\frac{E(k)}{4 \pi k^{2} \bar{e}(k)}, \\
\bar{e}(k)=1-\frac{v k^{2}}{\bar{\eta}(k)} .
\end{gathered}
$$

Equation (4.6) indicates that the shape of the energy spectrum $E(k)$ must be provided in order to determine the integral in (4.4). Qian (1986) used a functional form which combines the Kolmogorov spectrum with a small-scale correction. The large-scale behaviour is neglected. In the present analysis, equation (4.6) is determined using EDQNM spectra, which exhibit a very high resolution in the whole spectral domain of active scales. The investigation has been performed with thirty six different EDQNM spectra in the range $200 \leqslant R e_{\lambda} \leqslant 5 \times 10^{5}$, using the database for $\sigma=4$. For each configuration, the integral in (4.4) has been calculated using Monte Carlo integration with $10^{7}$ samples. The final integral was calculated by averaging the results of approximately 200 realisations of the integration in (4.6). The results are shown in figure 5 together with the analytic result of Qian (1986) and some experimental and DNS data available in the literature. The DNS data (Ishihara et al. 2007) include all values of $F$ for the various mesh resolutions and two wavenumber truncations (see earlier comments in $\S 3$ about these data).

\section{Strategy (ii)}

The result in figure 5, namely that $F$ approaches a plateau value, is used to perform an optimisation of the free coefficients in (2.8) and (2.9). It should be recalled that these free coefficients are derived from dimensional arguments. The values for $a_{\xi_{1}}$ and $a_{\xi_{2}}$ are optimised to obtain a fit (denoted EDQNM estimate in the figure) for $F=-(10 / 3) \gamma_{1}+(20 / 3)\left(\gamma_{2} / R e_{\lambda}\right)$ to match best the data of Qian (1986) for very 


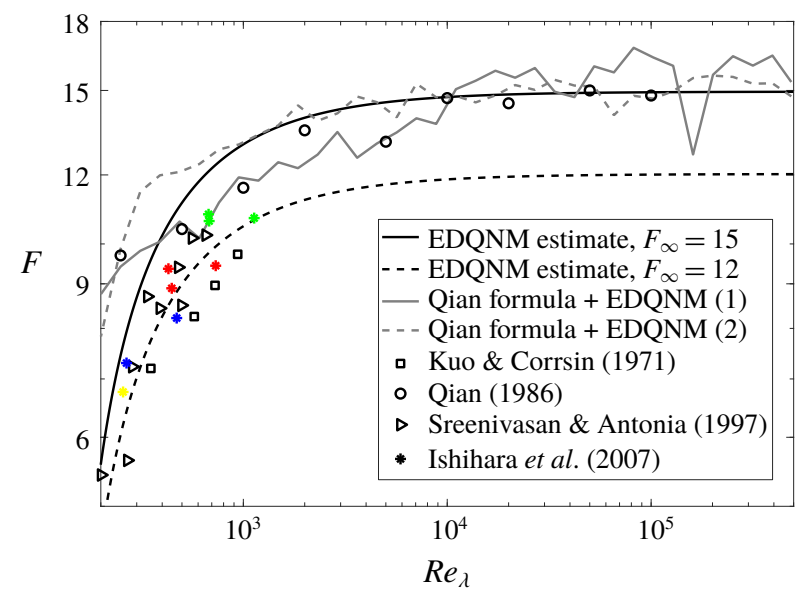

FIgURE 5. (Colour online) Velocity derivative flatness $F$ behaviour in the range of investigation of $R e_{\lambda}$. Data in the literature are shown along with an estimate reconstructed starting from EDQNM results. The two optimisation procedures target an asymptotic value of $F=15$ and $F=12$ for $R e_{\lambda} \rightarrow \infty$, respectively. Coloured symbols identify the different mesh resolutions of the DNS of Ishihara et al. (2007); yellow: 512 $2^{3}$, blue: $1024^{3}$, red: $2048^{3}$, green: $4096^{3}$.

high $R e_{\lambda}$ and experimental/numerical data in the literature (Kuo \& Corrsin 1971; Sreenivasan \& Antonia 1997; Ishihara et al. 2007) for moderate $R e_{\lambda}$. Two plateau values have been considered. $F_{\infty}=15$ which corresponds the asymptotic value found by Qian, and $F_{\infty}=12$, which according to a recent study by Tang et al. (2018) is a better asymptotic value for infinitely large Reynolds number. The final result, shown in figure 5, is consistent with the experimental and DNS data, giving confidence in the dimensional argument approach we used to assess the FRN effect on the terms of the expansion for $F$ and validating the results of figure 4 .

It is clear that the trend shown by the EDQNM data is consistent with the theoretical, experimental and DNS data: $F$ first increases as $R e_{\lambda}$ increases before approaching a plateau $F_{\infty}$ when $R e_{\lambda}$ becomes sufficiently large (e.g. $R e_{\lambda} \geqslant 10^{4}$ ). The important message of this figure is that while the actual value of the plateau is yet to be accurately determined, the present results are nevertheless of great interest because they reveal how $F$ evolves as $R e_{\lambda}$ increases. Further, even though the EDQNM simulation does not yield information about the actual asymptotic value for $F$ when $R e_{\lambda} \rightarrow \infty$, it shows that the behaviour exhibited by the DNS and experiment data when $R e_{\lambda}<1000$ is arguably affected, if not controlled, by FRN effects. Note that the constancy of $F$ at very large $R e_{\lambda}$ is fully consistent with the constancy of $S$ at similar $R e_{\lambda}$.

\subsection{Turbulent invariants}

The analysis in $\S 4.2$ shows how the coefficients $\gamma_{1}, \gamma_{2}$ and $\gamma_{3}$ exhibit a similar qualitative variation over the $R e_{\lambda}$ range investigated. We already mentioned that homogeneity imposes $\gamma_{2} \sim \gamma_{3}$. To investigate this, we report in figure 6 the ratio $\gamma_{2} / \gamma_{3}$ (imposing $a_{\xi_{2}}=a_{\xi_{3}}$ ). Further, the velocity derivative skewness $S$ is also shown in the figure for comparison. 


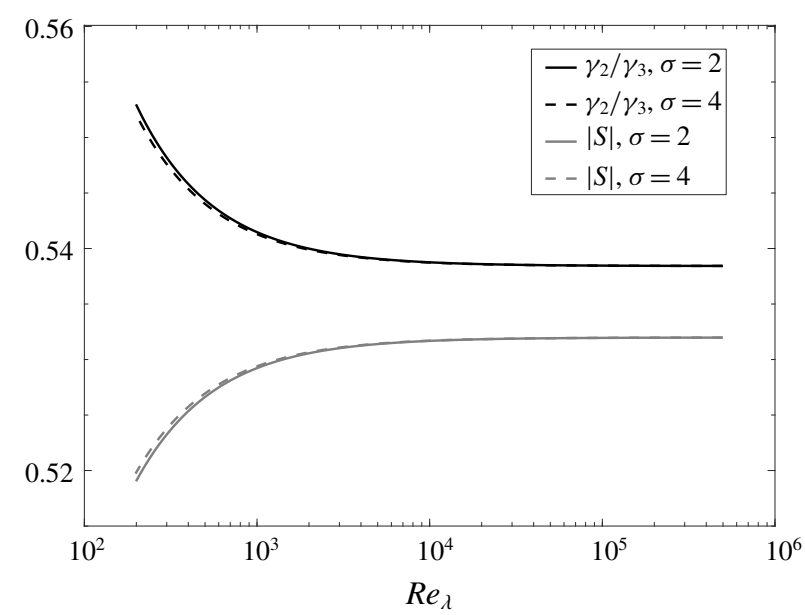

FIGURE 6. Evolution of the ratio $\gamma_{2} / \gamma_{3}$ compared with the velocity derivative skewness $S$. The results are reported for Saffman turbulence and Batchelor turbulence.

As expected, the ratio $\gamma_{2} / \gamma_{3}$ approaches a constant. Quite remarkably, the ratio $\gamma_{2} / \gamma_{3}$ exhibits a mirror-like behaviour to $|S|$. While this latter quantity increases with increasing $R e_{\lambda}$ before reaching a constant, $\gamma_{2} / \gamma_{3}$ decreases before becoming constant. Both quantities appear to reach a constant at the same rate as $R e_{\lambda}$ increases. Further, $\gamma_{2} / \gamma_{3}=0.538$ for high Reynolds numbers, which is very close to the asymptotic value $\left|S_{\infty}\right|=0.532$. The evolution of $\gamma_{2} / \gamma_{3}$ and $|S|$ suggest that an invariant can be defined as

$$
I_{\gamma}=\frac{\gamma_{2}}{\gamma_{3}} \frac{|S|}{S_{\infty}^{2}}
$$

and its variation with $R e_{\lambda}$ is reported in figure 7. $I_{\gamma} \approx 1.02$ for very high Reynolds numbers and is practically independent of $R e_{\lambda}$. Its variation is compared with that of:

(i) the velocity derivative skewness $S$;

(ii) the energy dissipation rate coefficient $C_{\epsilon}=(3 / 2)^{3 / 2}\left(\bar{\epsilon} L / \mathcal{K}^{3 / 2}\right)$ (Valente \& Vassilicos 2012; Djenidi et al. 2017c);

(iii) the length scale invariant $I_{L}=\mathcal{K} L^{\sigma+1}$, known as the Birkhoff-Saffman integral $I_{S}=\mathcal{K} L^{3}$ for Saffman turbulence and the Loytsianski integral $I_{B}=\mathcal{K} L^{5}$ for Batchelor turbulence. In the latter case, the integral including the correction for non-local interaction $I_{B 1}=\mathcal{K} L^{4.48}$ is considered as well (Meldi \& Sagaut 2012).

All of these quantities are supposed to be invariants for high Reynolds number free decaying HIT. Let us first analyse the case of Saffman turbulence, which is reported in figure $7(a)$. A zoom is provided in figure $7(c)$. The invariants are normalised by their asymptotic value at $\operatorname{Re}_{\lambda}=5 \times 10^{5}$. The coefficient exhibiting the largest variation over the investigation range is $C_{\epsilon}$, showing a decrease of approximately 30-35\%. This variation is associated with a lack of complete turbulence equilibrium due to the time evolution of the inertial range (Bos, Shao \& Bertoglio 2007). The Birkhoff-Saffman integral exhibits a much lower sensitivity to FRN effects. In particular, the evolution observed at very high Reynolds numbers is associated with minimal effects of initial conditions (see $n_{\mathcal{K}}$ in figure $3 b$ ). However, the parameter showing the most negligible variation is the invariant $I_{\gamma}$. Figure $7(c)$ shows that this parameter increases by just 
(a)

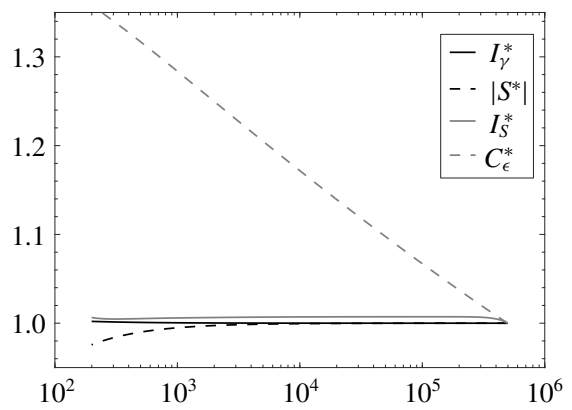

(c)

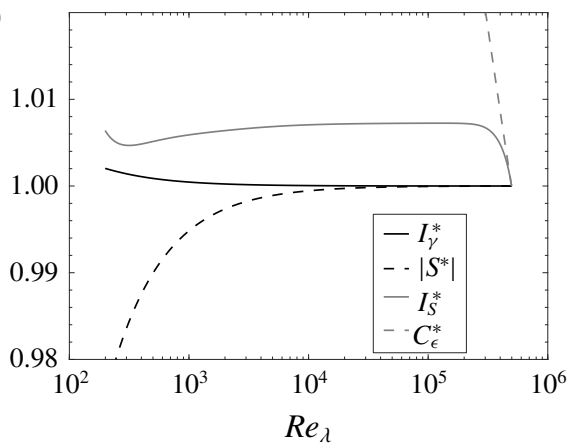

(b)

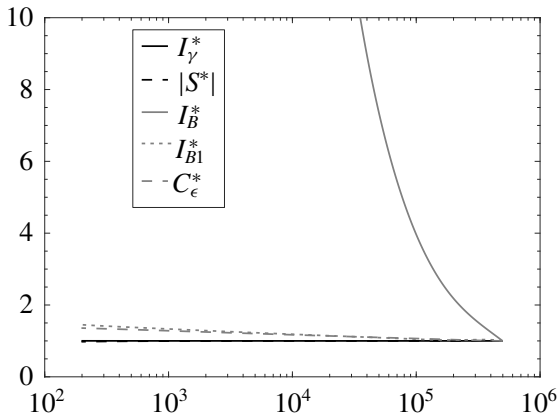

(d)

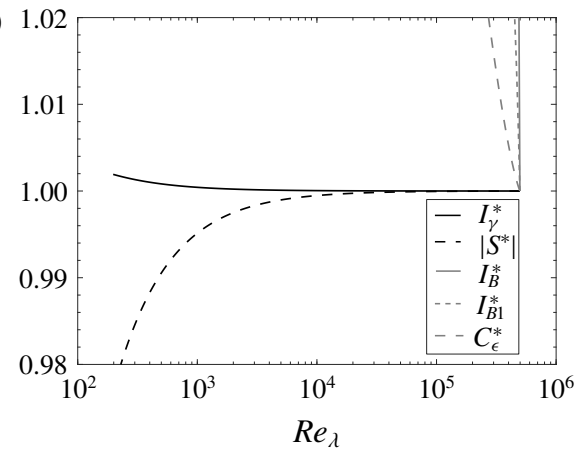

FIGURE 7. Evolution of turbulence invariants over the range of $R e_{\lambda}$ investigated. Results for $(a, c)$ Saffman turbulence and $(b, d)$ Batchelor turbulence are shown, respectively. All quantities are normalised by their respective value at the highest $\operatorname{Re}_{\lambda}$.

$0.2 \%$ over more than three decades of $R e_{\lambda}$. Similar considerations can be extended to the case of Batchelor turbulence, reported in figure $7(b, d)$. As already shown by Meldi \& Sagaut (2012) the classical Loytsianski integral is not an invariant, while the corrected integral $I_{B 1}$ exhibits a similar evolution as $C_{\epsilon}$. For Batchelor turbulence, the variation of $C_{\epsilon}$ is approximately $50 \%$, which is much larger than that for Saffman turbulence. This is associated with a stronger relaxation of the turbulence equilibrium condition. On the other hand, the Batchelor turbulence invariant $I_{\gamma}$ follows the same variation as that for Saffman turbulence, suggesting this could be a true (higher-order) universal invariant, which is also the case for $S$.

\section{Conclusions}

Numerical simulations based on a EDQNM model were carried out in freely decaying isotropic turbulence over more than three decades of the Taylor microscale Reynolds number, $\operatorname{Re}_{\lambda}\left(200 \leqslant R e_{\lambda} \leqslant 5 \times 10^{5}\right)$ in order to ascertain the effect of the Reynolds number on the skewness $S$ and flatness factor $F$ of the longitudinal velocity derivative. The choice of EDQNM is primarily based on the fact that it permits an investigation of the Reynolds number effect on statistical moments over a large range of $R_{\lambda}$. Since the moments of $\partial u / \partial x$ provide a measure of small-scale intermittency, the effect of the Reynolds number on the latter is investigated in a natural fashion, starting from the Navier-Stokes equations. Further, the present approach also avoids confinement effects that may for example exist in box turbulence simulations. While 
$S$ can be calculated directly from its definition, the effect of $R e_{\lambda}$ on $F$ is analysed via the analytic expression (2.4) (Djenidi et al. 2017b) through dimensional arguments where it is assumed that $F$ can be estimated dimensionally from only $k$ and $E(k)$ in similar fashion to the behaviour that can be determined via exact transport equations for the mean energy dissipation rate $\bar{\epsilon}$ and the palinstrophy $G$. Both Batchelor turbulence and Saffman turbulence are considered. The following results are found:

(i) $S$ reaches a constant value of approximately -0.53 ;

(ii) the first and second terms of the $F$-expression (2.4) become constant, indicating that $F$ too becomes constant. This result is also qualitatively consistent with that obtained by Qian (1986);

(iii) the constants mentioned above are reached for $R e_{\lambda} \geqslant 10^{4}$;

(iv) the EDQNM prediction (after optimising the free coefficients introduced by the dimensional arguments) of the behaviour of $F$ at moderate $R e_{\lambda}$ is consistent with the trend observed in the data reported in the literature, see Tang et al. (2018). The prediction also shows that $F$ approaches a constant when $R e_{\lambda} \rightarrow \infty$;

(v) the EDQNM calculation of the term $\int_{0}^{\infty} k^{* 4} E_{p}^{*}\left(k^{*}\right) \mathrm{d} k^{*}$, which Tang et al. (2018) identify as the upper limit for $F$, indicates that, as $R e_{\lambda}$ increases, this term first increases before becoming constant when $\operatorname{Re}_{\lambda} \geqslant 10^{4}$.

Further, a new invariant $I_{\gamma}$ is defined and compared with other classical invariants reported in the literature, such as the dissipation energy coefficient $C_{\epsilon}$, the BirkhoffSaffman integral and the Loytsianski integral. Over more than a three decade variation in $R e_{\lambda}, I_{\gamma}$ varies by approximately $0.2 \%$ and is independent of the large-scale features (irrespective of whether Saffman or Batchelor turbulence is used). This implies that, potentially, $I_{\gamma}$ is a true invariant, against which both experimental and numerical simulation results can be tested.

While the present analysis is carried out within the framework of EDQNM, the results raise some doubts regarding the anomalous behaviour usually associated with the effect of intermittency. Further, the results suggest that these effects, which are not accounted for by the EDQNM model, cannot be fully responsible for the trends observed in figure 5. Indeed, the FRN effect captured by the EDQNM results appears to be at least comparable with that derived from the Navier-Stokes equations, as reflected in the DNS and experimental data at moderate Reynolds numbers. One can argue that the absence of a model for intermittency (generally identified as the source of the Reynolds number dependence of $S$ and $F$ ) in the EDQNM model supports the view that the behaviour of $S$ and $F$ observed so far for $R e_{\lambda} \leqslant 10^{3}$ reflects a genuine FRN effect. It is important to stress that the present results do not imply a lack of intermittency. The results simply indicate that this phenomenon does not appear to control the behaviour of $S$ and $F$ for $R e_{\lambda} \leqslant 10^{3}$, which is the range of investigation achievable with experiments and DNS at the present time.

In conclusion, one must at least first ensure that $R e_{\lambda}$ is large enough for the FRN effect to be negligibly small before any anomalous behaviour due to intermittency can be assessed unambiguously.

\section{REFERENCES}

Antonia, R. \& Burattini, P. 2006 Approach to the 4/5 law in homogeneous isotropic turbulence. J. Fluid Mech. 550, 175-184.

Antonia, R., DJenidi, L., Danaila, L. \& TAng, S. 2017 Small scale turbulence and the finite Reynolds number effect. Phys. Fluids 29 (2), 020715. 
Antonia, R., Tang, S. L., Djenidi, L. \& Danaila, L. 2015 Boundedness of the velocity derivative skewness in various turbulent flows. J. Fluid Mech. 781, 727-744.

BATCHELOR, G. K. 1948 Energy decay and self-preserving correlation functions in isotropic turbulence. Q. Appl. Maths 6, 97-116.

Bos, W. J. T., Shao, L. \& Bertoglio, J. P. 2007 Spectral imbalance and the normalized dissipation rate of turbulence. Phys. Fluids 19 (4), 045101.

Comte-Bellot, G. \& Corrsin, S. 1966 The use of a contraction to improve the isotropy of grid-generated turbulence. J. Fluid Mech. 25, 657-682.

Danaila, L., Anselmet, F., Zhou, T. \& Antonia, R. A. 1999 A generalization of Yaglom's equation which accounts for the large-scale forcing in heated decaying turbulence. J. Fluid Mech. 391, 359-372.

Djenidi, L., Antonia, R. A., Talluru, M. K. \& Abe, H. $2017 a$ Skewness and flatness factors of the longitudinal velocity derivative in wall-bounded flows. Phys. Rev. Fluids 2 (6), 064608.

Djenidi, L., Danaila, L., Antonia, R. \& TAng, S. $2017 b$ A note on the velocity derivative flatness factor in decaying HIT. Phys. Fluids 29 (5), 051702.

Djenidi, L., Kamruzzaman, M. \& Antonia, R. 2015 Power-law exponent in the transition period of decay in grid turbulence. J. Fluid Mech. 779, 544-555.

Djenidi, L., Lefeuvre, N., Kamruzzaman, M. \& Antonia, R. 2017c On the normalized dissipation parameter $C_{\epsilon}$ in decaying turbulence. J. Fluid Mech. 817, 61-79.

Grossmann, S. \& Lohse, D. 1994 Scale resolved intermittency in turbulence. Phys. Fluids 6 (2), 611-617.

HILL, R. J. 2001 Equations relating structure functions of all orders. J. Fluid Mech. 434, 379-388.

Ishihara, T., Kaneda, Y., YokoKawa, M., ItakURA, K. \& UnO, A. 2007 Small-scale statistics in high-resolution direct numerical simulation of turbulence: Reynolds number dependence of one-point velocity gradient statistics. J. Fluid Mech. 592, 335-366.

Kolmogorov, A. N. 1941a The local structure of turbulence in incompressible viscous fluid for very large Reynolds number. Dokl. Akad. Nauk SSSR 30, 301-305; (see also Proc. R. Soc. Lond. A, 1991, 434, 9-13).

Kolmogorov, A. N. $1941 b$ Dissipation of energy in the locally isotropic turbulence. Dokl. Akad. Nauk SSSR 32, 16-18; (see also Proc. R. Soc. Lond. A, 1991, 434, 15-17).

Kolmogorov, A. N. 1962 A refinement of previous hypotheses concerning the local structure of turbulence in a viscous incompressible fluid at high Reynolds number. J. Fluid Mech. 13, $82-85$.

Kraichnan, R. H. 1974 On Kolmogorov's inertial-range theories. J. Fluid Mech. 62 (2), 305-330.

KUO, A. \& CORRSIN, S. 1971 Experiments on internal intermittency and fine-structure distribution functions in fully turbulent fluid. J. Fluid Mech. 50 (2), 285-319.

Landau, L. D. \& Lifshitz, E. M. 1987 Fluid mechanics: Vol. 6 of Course of Theoretical Physics, 2nd edn. Pergamon Press.

Lefeuvre, N., DJenidi, L. \& Antonia, R. A. 2015 Decay of mean energy dissipation rate on the axis of a turbulent round jet. In Proceedings of the Ninth Symposium on Turbulence and Shear Flow Phenomena (TSFP-9), 30 June-3 July, Melbourne, http://www.tsfp-conference.org/proceedings/tsfp9-contents-of-volume-3.html.

LESIEUR, M. 1997 Turbulence in Fluids, 3rd edn. Kluwer.

LindBorg, E. 1999 Correction to the four-fifths law due to variations of the dissipation. Phys. Fluids 11 (3), 510-512.

Lundgren, T. S. 2002 Kolmogorov two-thirds law by matched asymptotic expansion. Phys. Fluids 14 (2), 638-642.

MсСомв, W. D. 2014 Homogeneous, Isotropic Turbulence, Phenomenology, Renormalization and Statistical Closures. Oxford University Press.

Meldi, M. 2016 The signature of initial production mechanisms in isotropic turbulence decay. Phys. Fluids 28, 035105.

Meldi, M. \& SAGAUT, P. 2012 On non-self-similar regimes in homogeneous isotropic turbulence decay. J. Fluid Mech. 711, 364-393. 
Meldi, M. \& Sagaut, P. $2013 a$ Further insights into self-similarity and self-preservation in freely decaying isotropic turbulence. J. Turbul. 14, 24-53.

Meldi, M. \& Sagaut, P. $2013 b$ Pressure statistics in self-similar freely decaying isotropic turbulence. J. Fluid Mech. 717, R2.

Meldi, M. \& Sagaut, P. 2014 An adaptive numerical method for solving EDQNM equations for the analysis of long-time decay of isotropic turbulence. J. Comput. Phys. 262, 72-85.

Meldi, M. \& Sagaut, P. 2017 Turbulence in a box: Quantification of large-scale resolution effects in isotropic turbulence free decay. J. Fluid Mech. 818, 697-715.

Meyers, J. \& Meneveau, C. 2008 A functional form for the energy spectrum parametrizing bottleneck and intermittency effects. Phys. Fluids 20 (6), 065109.

MYdLARSKI, L. \& WARHAFT, Z. 1996 On the onset of high- Reynolds-number grid-generated wind tunnel turbulence. J. Fluid Mech. 320, 331-368.

Овоuкhov, А. 1962 Some specific features of atmospheric turbulence. J. Fluid Mech. 13 (1), 77-81.

Orszag, S. A. 1970 Analytical theories of turbulence. J. Fluid Mech. 41, 363-386.

Pope, S. B. 2000 Turbulent Flows. Cambridge University Press.

QIAN, J. 1982 Variational approach to the closure problem of turbulence theory. Phys. Fluids 26 (8), 2098-2104.

QIAN, J. 1986 A closure theory of intermittency of turbulence. Phys. Fluids 29 (7), 2165-2171.

QIAN, J. 1994 Skewness factor of turbulent velocity derivative. Acta Mechanica Sin. 10, 12-15.

QIAN, J. 1997 Inertial range and the finite Reynolds number effect of turbulence. Phys. Rev. E 55 (1), 337.

QIAN, J. 1999 Slow decay of the finite Reynolds number effect of turbulence. Phys. Rev. E 60 (3), 3409.

QIAN, J. 2000 Closure approach to high-order structure functions of turbulence. Phys. Rev. Lett. 84 (4), 646.

SAfFMAn, P. J. 1967 The large-scale structure of homogeneous turbulence. J. Fluid Mech. 27, 581-593.

Sagaut, P. \& Cambon, C. 2018 Homogenous Turbulence Dynamics. Springer.

Sreenivasan, K. \& Antonia, R. A. 1997 The phenomenology of small-scale turbulence. Annu. Rev. Fluid Mech. 29, 435-472.

Tang, S., Antonia, R., Djenidi, L., Abe, H., Zhou, T., Danaila, L. \& Zhou, Y. $2015 a$ Transport equation for the mean turbulent energy dissipation rate on the centreline of a fully developed channel flow. J. Fluid Mech. 777, 151-177.

Tang, S., Antonia, R., DJenidi, L., Danaila, L. \& Zhou, Y. 2017 Finite Reynolds number effect on the scaling range behaviour of turbulent longitudinal velocity structure functions. J. Fluid Mech. 820, 341-369.

Tang, S., Antonia, R., Djenidi, L., Danaila, L. \& Zhou, Y. 2018 Reappraisal of the velocity derivative flatness factor in various turbulent flows. J. Fluid Mech. 847, 244-265.

TAng, S., Antonia, R., DJenidi, L. \& Zhou, Y. 2015b Transport equation for the isotropic turbulent energy dissipation rate in the far-wake of a circular cylinder. J. Fluid Mech. 784, $109-129$.

Tchoufag, J., Sagaut, P. \& CAmbon, C. 2012 Spectral approach to finite Reynolds number effects on Kolmogorovs 4/5 law in isotropic turbulence. Phys. Fluids 24 (1), 015107.

VAlente, P. C. \& VAssilicos, J. C. 2012 Dependence of decaying homogeneous isotropic turbulence on inflow conditions. Phys. Lett. A 376, 510-514.

Zhou, T., Antonia, R., Danaila, L. \& Anselmet, F. 2000 Approach to the four-fifths 'law' for grid turbulence. J. Turbul. 1, N5.

Zhou, T., Antonia, R. A. \& ChuA, L. P. 2005 Flow and Reynolds number dependencies of one-dimensional vorticity fluctuations. J. Turbul. 6, 1-17. 